\title{
Clinicopathological Significance of CD133 and ALDH1 Cancer Stem Cell Marker Expression in Invasive Ductal Breast Carcinoma
}

\author{
Sahar F Mansour, Maha M Atwa*
}

\begin{abstract}
Background: Biomarkers in breast neoplasms provide invaluable information regarding prognosis and help determining the optimal treatment. We investigated the possible correlation between cancer stem cell (CSC) markers (CD133, and ALDH1) in invasive ductal breast carcinomas with some clinicopathological parameters. Aim: To assess the correlation between expression of cancer stem cell (CSC) markers (CD133, and ALDH1) and clinicopathological parameters of invasive ductal breast carcinomas. Materials and Methods: Immunohistochemical analysis of CD133 and ALDH1 was performed on a series of 120 modified radical mastectomy (MRM) specimens diagnosed as invasive ductal breast carcinoma. Results: Expression of both CD133 and ALDH1 was significantly changed and related to tumor size, tumor stage (TNM), and lymph node metastasis. A negative correlation between CD133 and ALDH1 was found. Conclusions: Detecting the expression of CD133 and ALDH1 in invasive ductal breast carcinomas may be of help in more accurately predicting the aggressive properties and determining the optimal treatment.
\end{abstract}

Keywords: Breast carcinoma - cancer stem cell markers - CD133 - ALDH1

Asian Pac J Cancer Prev, 16 (17), 7491-7496

\section{Introduction}

Breast cancer is the most common primary site in women (30\% on average), followed by colorectal (13\%), lung $(8 \%)$, and cervical carcinoma $(5 \%)$ and the most likely cause that a woman will die from cancer worldwide (Ferlay et al., 2013).

According to official statistics of the National Cancer Institute (Cairo University), breast cancer accounts for $35.1 \%$ of the cases of cancer in Egypt and is the most prevalent cancer among Egyptian women (Ibrahim et al., 2014).

In spite of advances in diagnosis and treatment of breast cancer, almost one-fourth of women with this neoplasm will die. The major causes of treatment failure and/or death for patients with breast neoplasms are tumor invasion and metastasis. Decisions regarding the use of adjuvant and palliative therapies in patients with breast neoplasms rely primarily on prognostic factors, such as tumor grade and size, axillary nodal status, distant metastasis (Lamy et al., 2013) and candidate biomarkers, such as hormone receptor (estrogen receptor (ER) and progesterone receptor (PR) expression, and c-erbB2/Her-2/neu) amplification/ overexpression. Therefore, such biomarkers in breast neoplasms provide information regarding the outcome of patients (Tessari et al., 2013; Turner et al., 2014). A study in search of additional biomarkers is necessary for patients with breast neoplasms.
The cancer stem cell model suggests that in many cancers, tumor initiation and propagation is driven by a population of self-renewing tumor cells known as cancer stem cells (CSCs) (Reya, et al., 2001). CSCs also promote tumor cell heterogeneity, metastasis, and therapeutic resistance, and are potentially driven by known oncogenic signaling pathways. The study of CSCs would be greatly enhanced by the availability of specific markers to identify and isolate these cells (Meacham and Morrison, 2013). Through examinations using putative stem cell markers or side population, unique subsets of cancer cells from different types of tumors have been detected. These markers include CD133, CD44, CD24, and CD166. Among them, both CD133 and CD44 are widely used for isolating CSCs from solid tumors (Clark and Fuller, 2006; Visvader and Lindeman, 2008; Navin et al., 2011).

CD133 is a glycoprotein also known in humans and rodents as Prominin 1 (PROM1). It is a member of pentaspan transmembrane glycoproteins (Bertolini et al., 2009). CD133 is expressed in hematopoietic stem cells (Timothy et al., 2013), endothelial progenitor cells (Liao et al., 2010), gastric cancer (Saricanbaz et al., 2014), glioblastoma (Yan et al., 2011), neuronal and glial stem cells (Bexell et al., 2009), squamous cell carcinoma (Satpute et al., 2013) various pediatric brain tumors (Rasha et al., 2012) as well as adult kidney, mammary glands, trachea, salivary glands, placenta, digestive tract, testes, and some other cell types (Tirino et al., 2009; Shi et al., 
2010; Bozzi et al., 2011).

Aldehyde dehydrogenase 1 (ALDH1) is an enzyme having the role of oxidating intracellular aldehydes and that could have the responsibility to oxidize retinol to retonic acid in stem cells (Jiang et al., 2009; Alison et al., 2010). Sorting cells based on high ALDH activity has been shown to enrich for CSC in several cancers (Douville et al., 2009), including carcinoids (Gaur et al., 2011). The use of ALDH1 as a target molecule to select cancer stem cells has been facilitated by a combination of live cell ALDH1 activity detection and cell sorting techniques that proposed it as a diagnostic marker, and a therapeutic target as well as a prognostic marker in a number of cancers including cervical carcinoma (Rao et al., 2012) and urothelial carcinoma of the urinary bladder (Keymoosi et al., 2014).

In this study, we evaluated the expression and distribution of the representative CSC markers CD133 and ALDH1 in breast cancer by immunohistochemistry, and studied their relationship with clinicopathologic features. We studied the interrelationship between the expressions of these two proteins.

\section{Materials and Methods}

\section{Tissue samples}

One hundred twenty patients who underwent MRM for treatment of invasive ductal breast carcinomas between years 2006-2013 at the Suez Canal University Hospital were retrospectively included in this study. No patients had evidence of distant metastasis at the time of primary surgery based on the preoperative examination. Tumor tissue specimens were retrieved from the archives of the Department of Pathology. The slides were reviewed by the researchers to ensure that the cases were consistent with breast ductal carcinoma. Clinical information, tumor size, and axillary lymph node status were obtained from medical records and the pathology reports.

\section{Immunohistochemical examination}

All samples were fixed in $10 \%$ buffered formalin and embedded in paraffin. Four- micrometer thick tissue sections were used for analysis. All sections were deparaffinized and dehydrated with graded alcohol. Then, the sections were washed for ten minutes in PBS at $\mathrm{pH}$ 7.2. The endogenous peroxidase activity was quenched by incubation in methanol containing $3 \% \mathrm{H} 2 \mathrm{O} 2$ for ten minutes at room temperature, then heated for 30 minutes at $95^{\circ} \mathrm{C}$ to repair the antigens and finally rinsed in PBS. After several washes in PBS, sections were blocked with goat serum for 20 minutes at room temperature, and then incubated with mouse monoclonal CD133 (Santa Cruz Biotechnology Inc., Santa Cruz, CA, USA) and anti-ALDH1 antibody (Santa Cruz Biotechnology, INC) 1:200 primary antibodies overnight at $4^{\circ} \mathrm{C}$ in a humidified chamber. The slides were treated with polymer enhancer (reagent A) for 20 minutes at room temperature. Washing in PBS, the slides were treated with goat anti-mouse antibody (reagent B) for 30 minutes at room temperature. After a complete wash in PBS, the slides were developed in freshly prepared diaminobenzidine (DAB) solution for eight minutes, and then counterstained with hematoxylin, dehydrated, air-dried, and mounted.

Serial sections of invasive duct carcinoma were run in parallel with the primary antibody replaced by PBS and rabbit IgG1 as blank and negative controls.

\section{Evaluation of immunohistochemistry}

Antigen expression was evaluated independently by the two authors using light microscopy. Both assessors were unaware of the clinical outcome. Equivocal cases were re-assessed on a double-headed microscope to establish a final score. For each sample, at least five fields (inside the tumor and in the area exhibiting tumor invasion; $\times 400$ ) were analyzed. Scores were applied as follows: score 0 , negative staining in all cells; score $1+$, weakly positive or focally positive staining in $<10 \%$ of the cells; score $2+$, moderately positive staining covering $10 \%$ to $50 \%$ of the cells; and score $3+$, strongly positive staining, including $>50 \%$ of the cells. For statistical analysis, as well as to reduce intraobserver variability, the immunohistochemical scores were further grouped into two categories: negative or weakly positive $(0$ and $1+)$ and moderately-to-strongly positive (2+ and $3+$ ).

The positive expression of CD133 was found mainly on the membrane and cytoplasm of tumor cells. The positive expression of ALDH1was found mainly on the membrane and cytoplasm of tumor cells. They were presented as a brown granular material.

\section{Statistical analysis}

Fisher's exact test and Spearman's correlate analysis for univariate or multivariate analysis were used to assess the associations among the positive staining of CD133 or ALDH1 and clinicopathological indices. SPSS 16.0 software for windows (Chicago, IL, USA) was used for this purpose. A value of $\mathrm{P}<0.05$ was considered statistically significant.

Table 1. Clinicopathological Characteristics of the Patients and Tumors

\begin{tabular}{lcc}
\hline Characteristic & $\mathrm{n}$ & $(\%)$ \\
\hline Mean age \pm SD, yrs (range), & $49.1 \pm 12.7$ & $28-70 \mathrm{yrs}$ \\
Histologic grade (Nottingham Histologic score) & \\
$\quad$ Grade I & 48 & $(40)$ \\
$\quad$ Grade II & 56 & $(46.7)$ \\
Grade III & 16 & $(13.3)$ \\
Tumor size (pT) & & \\
pT1 & 48 & $(40)$ \\
pT2 & 56 & $(46.7)$ \\
pT3 & 12 & $(10)$ \\
p T4 & 4 & $(3.3)$ \\
Regional Lymph node metastasis (pN) & & \\
Negative (pN0) & 40 & $(33.3)$ \\
Positive & 80 & $(66.7)$ \\
Stage (TNM) & & \\
Stage I & 40 & $(33.3)$ \\
Stage II & 64 & $(53.3)$ \\
Stage III & 16 & $(13.3)$ \\
Ductal carcinoma in situ component (DCIS) & \\
Minor (less than 25\%) & 80 & $(66.7)$ \\
Extensive (More than 25\%) & 40 & $(33.3)$ \\
\hline
\end{tabular}




\section{Results}

The age of the patients at time of diagnosis was ranged from 28 to 71 years, and the median age was 49 years. No patients had evidence of distant metastasis at the time of surgery. The majority of cases $64(53.3 \%)$ had stage II disease and the tumor diameter between 2 and $5 \mathrm{~cm}$ was detected in $56(46.7 \%)$ of the patients. The details of patient characteristics and descriptive statistics for the tumors are shown in Table 1.

\section{Immunohistochemical staining}

CD133 was expressed in the tumor cell membrane and the cytoplasm of cancer cells (Figure A). Expression of CD133 in tumor cells occurred in $53.3 \%$ (64/120) of the cases. As shown in Table 2, CD133 expression was significantly correlated with some clinicopathological parameters, including age $(\mathrm{p}=0.0435)$, tumor size $(\mathrm{pT})$ $(\mathrm{P}=0.0031)$, lymph node metastasis $(\mathrm{P}=0.0196)$ and highly statistically significant with tumor stage (TNM) $(\mathrm{P}=0.0001)$. CD133 was mainly expressed in larger tumors, lymph node metastasis and advanced tumor stage, indicating CD133 related to more aggressive characters of tumor. However, there was no significant correlation of CD133 expression with histopathological grade (Nottingham Histologic score) and Insitu Ductal Carcinoma component percentage.

Out of 120 cases of invasive ductal breast carcinoma, 50 cases $(41.7 \%)$ were positive for ALDH1. ALDH1 was observed mainly in the cytoplasm and the membrane

Table 2. Correlations between CD133 or ALDH1 Expression and Clinicopathological Parameters

\begin{tabular}{|c|c|c|c|c|c|c|c|c|c|}
\hline \multirow[t]{3}{*}{ Variable } & \multirow{3}{*}{$\begin{array}{c}\text { Number of } \\
\text { Patients }\end{array}$} & \multicolumn{4}{|c|}{ CD133 expression } & \multicolumn{4}{|c|}{ ALDH-1 expression } \\
\hline & & $\begin{array}{c}\text { Positive, } \\
(\mathrm{n}=64)\end{array}$ & \multicolumn{2}{|c|}{$\begin{array}{c}\text { Negative, } \\
(\mathrm{n}=56)\end{array}$} & \multirow[t]{2}{*}{ P value } & $\begin{array}{c}\begin{array}{c}\text { Positive, } \\
(\mathrm{n}=50)\end{array}\end{array}$ & \multicolumn{2}{|c|}{$\begin{array}{c}\text { Negative, } \\
(\mathrm{n}=70)\end{array}$} & \multirow[t]{2}{*}{$\mathrm{P}$ value } \\
\hline & & $\mathrm{n} \quad(\%)$ & & $(\%)$ & & $\mathrm{n} \quad(\%)$ & $\mathrm{n}$ & $(\%)$ & \\
\hline \multicolumn{10}{|l|}{ Age } \\
\hline$<49$ & 56 & $24(20)$ & 32 & (27) & \multirow[t]{2}{*}{$0.0435^{*}$} & $20(17)$ & 36 & (30) & \multirow[t]{2}{*}{0.2664} \\
\hline$>49$ & 64 & $40(33)$ & 24 & $(20)$ & & $30(25)$ & 34 & (20) & \\
\hline \multicolumn{10}{|c|}{ Tumor size (pT) } \\
\hline pT1-pT2 & 104 & $50(42)$ & 54 & $(45)$ & \multirow[t]{2}{*}{$0.0031 *$} & 35 (29) & 69 & $(57)$ & \multirow[t]{2}{*}{$0.0001 *$} \\
\hline pT3-pT4 & 16 & $14(11)$ & 2 & (2) & & $15(13)$ & 1 & (1) & \\
\hline \multicolumn{10}{|c|}{ Histologic grade (Nottingham Histologic score) } \\
\hline I & 48 & $24(20)$ & 24 & $(20)$ & \multirow[t]{2}{*}{0.5796} & $15(12)$ & 33 & $(28)$ & \multirow[t]{2}{*}{0.0627} \\
\hline II-III & 72 & $40(33)$ & 32 & $(27)$ & & $35(29)$ & 37 & $(31)$ & \\
\hline \multicolumn{10}{|c|}{ Lymph node metastasis $(\mathrm{pN})$} \\
\hline Negative & 40 & $15(12)$ & 25 & $(21)$ & \multirow[t]{2}{*}{$0.0196 *$} & $10 \quad(9)$ & 30 & $(25)$ & \multirow[t]{2}{*}{$0.0107^{*}$} \\
\hline Positive & 80 & $49(41)$ & 31 & (26) & & $40(33)$ & 40 & $(33)$ & \\
\hline \multicolumn{10}{|c|}{ Stage (TNM) } \\
\hline I & 40 & $10 \quad(9)$ & 30 & $(25)$ & \multirow[t]{2}{*}{$0.0001 *$} & $16(14)$ & 24 & $(20)$ & \multirow[t]{2}{*}{0.846} \\
\hline II-III & 80 & $52(43)$ & 28 & $(23)$ & & $34(28)$ & 46 & $(38)$ & \\
\hline \multicolumn{10}{|c|}{ Ductal carcinoma in situ component (DCIS \%) } \\
\hline$<25 \%$ & 80 & $42(35)$ & 38 & $(32)$ & \multirow[t]{2}{*}{0.8476} & $29(24)$ & 51 & $(43)$ & \multirow[t]{2}{*}{0.1163} \\
\hline & 40 & $22(18)$ & 18 & $(15)$ & & $21(17)$ & 19 & $(16)$ & \\
\hline
\end{tabular}

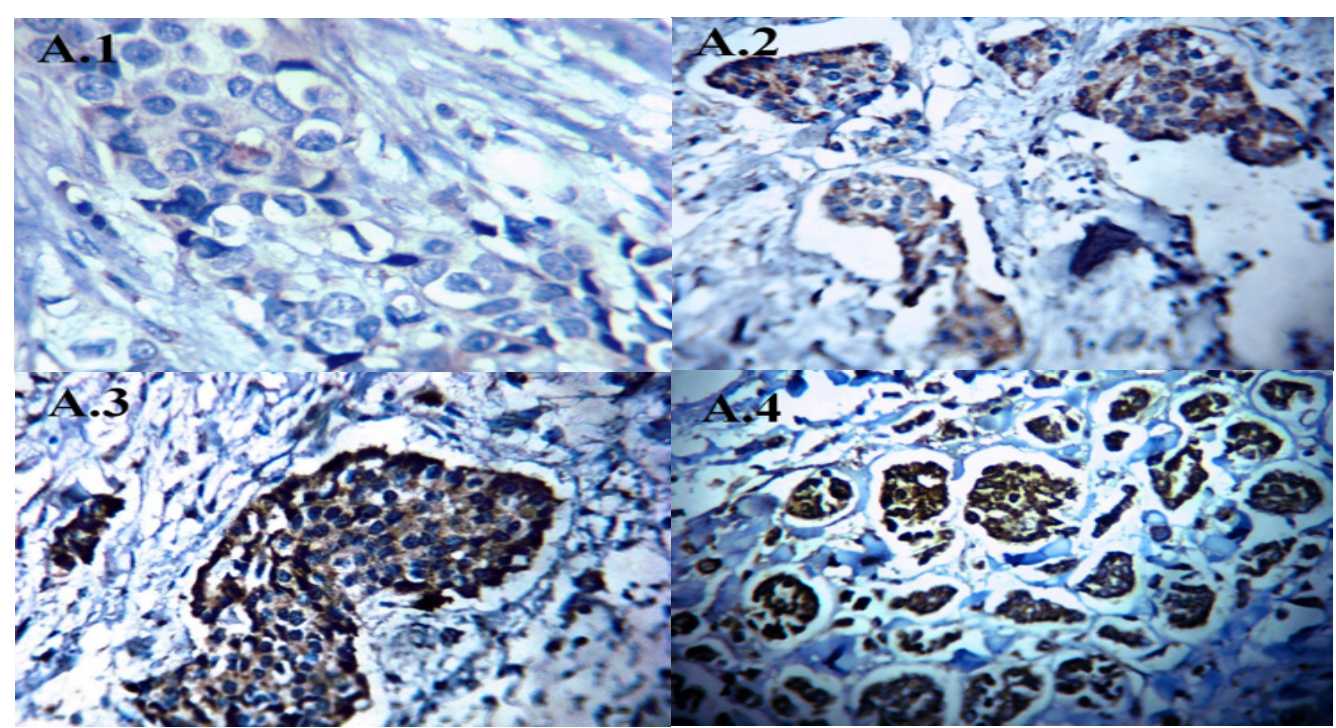

Figure 1A. Immunohistochemical Determination of CD133 Expression. The CD133 Antibody Stained Intensely at the Membrane and in the Cytoplasm of Cancer Cells. Scores were applied as follows: Score 0: negative staining in all cells; Score 1+: weakly positive or focally positive staining in $<10 \%$ of cells (Figure A.1); Score $2+$, moderately positive staining in $10 \%-$ $50 \%$ of cells (Figure A.2); Score $3+$, strongly positive-staining, involving 50\% or more of the cells (Figure A.3 and Figure A.4) 


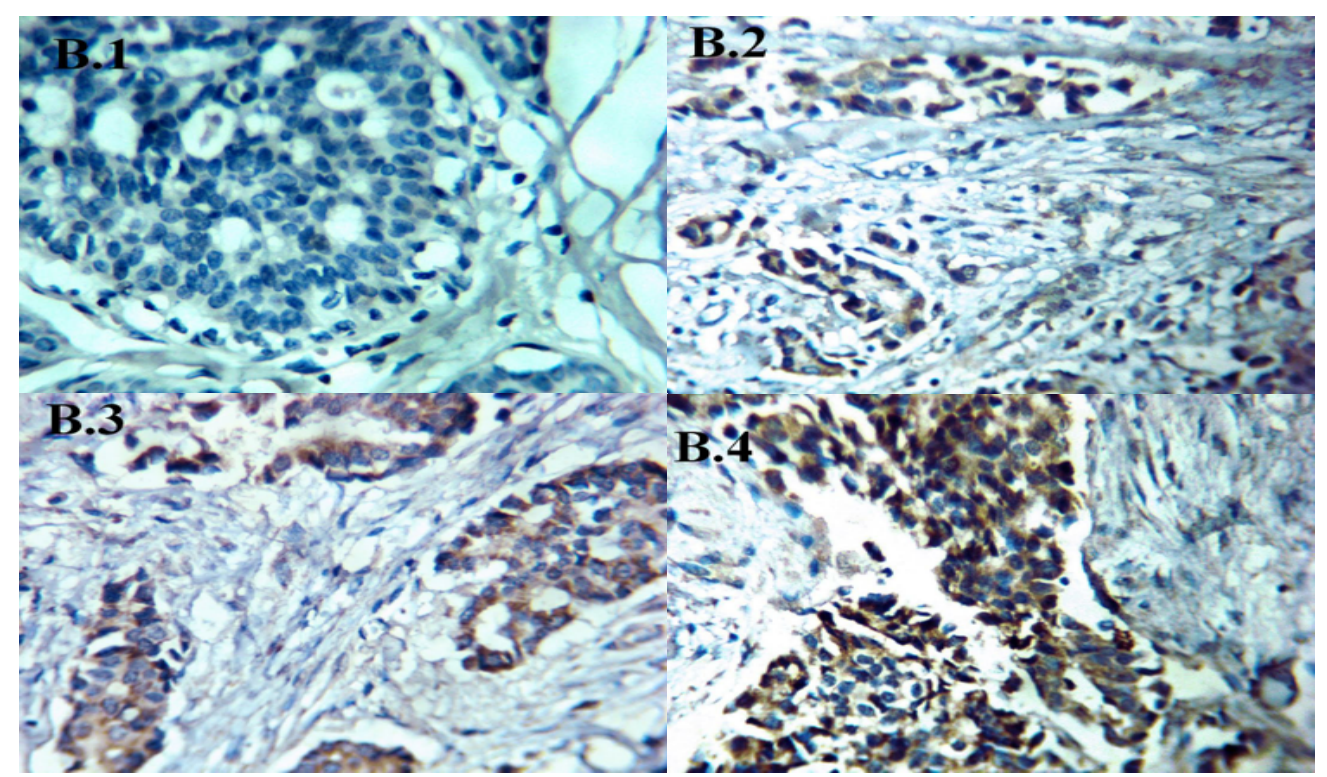

Figure 2B. Immunohistochemical Determination of ALDH1 Expression. The ALDH1 antibody stained intensely at the membrane and in the cytoplasm of cancer cells. Scores were applied as follows: Score 0: negative staining in all cells; Score 1+: weakly positive or focally positive staining in $<10 \%$ of cells (Figure B.1); Score $2+$, moderately positive staining in $10 \%-50 \%$ of cells (Figure B.2); Score 3+, strongly positive-staining, involving 50\% or more of the cells (Figure B.3 and Figure B.4)

of the tumor cells (Figure B). ALDH1 expression was significantly correlated with some clinicopathological parameters including tumor size $(\mathrm{pT})(\mathrm{p}=0.0001)$, lymph nodes metastasis $(\mathrm{p}=0.0107)$. Although not significantly correlated ALDH1-positive cases are seen more with high histological grade ( $\mathrm{p}=0.0627)$, advanced TNM stage $(\mathrm{P}=0.8460)$, minor DCIS \% $(\mathrm{P}=0.1163)$ than ALDH1negative cases (table 2).

\section{Discussion}

According to the CSC hypothesis, it is assumed that CSCs are responsible for cancer initiation and development. Expression of CSC markers has been reported to be present in normal adult stem/ progenitor cells as well as in CSC (Clevers (2011); Mills and Shivdasani, 2011). In the present study, we examined the expression and distribution of representative CSC markers (CD133 and ALDH1) in ductal carcinoma of the breast, the most common subtype of breast carcinomas. Originally considered a marker of hematopoietic stem cells, CD133/ prominin is a highly glycosylated trans-membrane protein expressed in various tissues, such as breast, in which it seems to regulate ductal branching but not regenerative capacity (Zobalova et al., 2011). The protein CD133 is one of the hot CSC markers in a variety of tumors (Wang et al., 2011; Zhao et al., 2011; Di Bonito et al., 2012; Schneider et al., 2012). In this study, we found that the positive expression of CD133 was 53.3\% (64/120) in invasive duct carcinoma and it was significantly correlated with some clinicopathological parameters, including age $(\mathrm{p}=0.0435)$, tumor size (pT) $(\mathrm{P}=0.0031)$, lymph node metastasis $(\mathrm{P}=0.0196)$ and highly significant with the tumor stage (TNM) $(\mathrm{P}=0.0001)$. CD133 was mainly expressed in larger tumors, lymph node metastasis and advanced tumor stage, indicating CD133 related to more aggressive characters of tumor. However, there was no significant correlation of CD133 expression with histopathological grade (Nottingham Histologic score) and Insitu Ductal Carcinoma component. As CD133 expression was more in more aggressive characters of tumor (higher stage tumors, larger tumors, and lymph node metastasis), suggesting the expression of CD133 might be a potential prognostic factor in invasive duct carcinoma of the breast. These results are consistent with previous reports that CSC marker expression is significantly upregulated in some solid carcinomas and are risk factors for worse clinical behavior (Shimada et al., 2009; Charafe-Jauffret et al., 2010). However, there was no significant correlation of CD133 expression with age, histopathological grade and Insitu Ductal Carcinoma component. Recent and numerous studies show that positivity for CD133 allows identifying CSCs in breast cancer (Wright et al., 2008). CD133 is expressed by several solid tumors, including invasive breast cancer triple negative, with very low levels of expression compared to other CSCs markers previously reported, like CD44 and ALDH1 (Wu and Wu, 2009). In early-onset breast cancers, associated with mutations on BRCA1, CD133+ cells show CSCs properties (Wright et al., 2008). The employment of this tumor stemness marker in breast cancers has become popular more recently and its expression is often described as associated with a worse prognosis (Ieni et al., 2011; Zhao et al., 2011).

The breast CSC marker ALDH1 has been described as a marker of both normal and malignant breast stem/ progenitor cells (Huang et al., 2009; Meyer et al., 2009; Deng et al., 2010). ALDH1hi tumor cells form visibly larger colonies and mammospheres, when compared with ALDH1low cells (Deng et al., 2010). Previous works also detected small percentages of ALDH1+ cases in invasive breast cancer, ranging from $4 \%$ to $19 \%$ (Morimoto et al., 2009; Park et al., 2010; Resetkova et al., 2010). In our study, we found $42 \%(50 / 120)$ of ALDH1 expression. ALDH1 expression was significantly correlated with some clinicopathological parameters including tumor size $(\mathrm{pT})(\mathrm{p}=0.0001)$, and lymph nodes metastasis 
$(\mathrm{p}=0.0107)$. Although not significantly correlated ALDH1positive cases were seen more with high histological grade $(\mathrm{p}=0.0627)$, advanced TNM stage $(\mathrm{P}=0.8460)$, or minor DCIS \% $(\mathrm{P}=0.1163)$ than ALDH1-negative cases. These results are consistent with previous reports that ALDH1 expression in breast cancer accounts for $20-50 \%$. High positivity in tumor cells is associated with high histological grade, ERBB2 over-expression, absence of hormone receptors ER and $\mathrm{PgR}$ and worse prognosis (Ohi et al., 2011; Sakakibara et al., 2012). However the study of (Madjd et al., 2012) couldn't find statistical correlation between ALDH1 expression and breast tumor characteristics except a noticeable trend relation between ALDH1 expression and high grade tumors.

CD133 and ALDH1 expression, which could be detected by immunohistochemistry, might be a useful molecular marker to predict the prognosis in invasive ductal carcinoma of breast patients. The current study concluded that the expression of CD133 and ALDH1 proteins could be correlated with lymph node metastasis, grade of tumor, and pTNM stage in invasive ductal carcinoma of breast. The combined detection of CD133 and ALDH1 can, to some extent, reflect the biological behavior of invasive ductal carcinoma of breast, thus giving the choice of molecular targeting therapy.

In conclusion, It is suggested that CD133 and ALDH1 may play an important role in the evolution of invasive ductal carcinoma of breast and CD133 along with ALDH1 should be considered as potential marker for the prognosis in patients with invasive ductal carcinoma.

\section{References}

Alison MR, Guppy NJ, Lim SM, et al (2010). Finding cancer stem cells, are aldehyde dehydrogenases fit for purpose?. $J$ Pathol, 222, 335-44.

Bertolini G, Roz L, Perego P, et al (2009). Highly tumorigenic lung cancer CD133+ cells display stem-like features and are spared by cisplatin treatment. Proc Natl Acad Sci U.S.A, 106, 16281-6.

Bexell D, Gunnarsson S, Siesjö P, et al (2009). CD133+ and nestin+ tumor-initiating cells dominate in N29 and N32 experimental gliomas. International J Cancer, 125, 15-22.

Bozzi F, Conca E, Manenti G, et al (2011). High CD133 expression levels in gastrointestinal stromal tumors. Cytometry B Clin Cytom, 80, 238-47.

Charafe-Jauffret E, Ginestier C, Iovino F, et al (2010). Aldehyde dehydrogenase 1-positive cancer stem cells mediate metastasis and poor clinical outcome in inflammatory breast cancer. Clin Cancer Res, 16, 45-55.

Clark MF, and Fuller M (2006). Stem cells and cancer, Two faces of eve. Cell, 124, 1111-5.

Clevers H (2011). The cancer stem cell, Premises, promises and challenges. Nat Med, 17, 313-9.

Deng S, Yang X, Lassus H, et al (2010). Distinct expression levels and patterns of stem cell marker, aldehyde dehydrogenase isoform 1 (ALDH1), in human epithelial cancers. PLoS One, 5, 10277.

Di Bonito M, Collina F, Cantile M, et al (2012). Aberrant expression of cancer stem cells marker prominin- 1 in low-grade tubulobular breast carcinoma, a correlative study between qRT-PCR, Flow-Cytometric and Immunohistochemistry Analysis. J Breast Cancer, 15, 15-23.
Douville J, Beaulieu R \& Balicki D (2009). ALDH1 as a functional marker of cancer stem and progenitor cells. Stem Cells Dev, 18, 17-25.

Ferlay J, Soerjomataram I, Ervik M, et al (2013). Cancer Incidence and Mortality Worldwide (2013). IARC Cancer Base No. 11 Lyon, France, International Agency for Research on Cancer. GLOBOCAN v1.0

Gaur P, Sceusi EL, Samuel S, et al (2011). Identification of cancer stem cells in human gastrointestinal carcinoid and neuroendocrine tumors. Gastroenterology, 141, 1728-37.

Huang EH, Hynes MJ, Zhang T, et al (2009). Aldehyde dehydrogenase 1 is a marker for normal and malignant human colonic stem cells (SC) and tracks SC overpopulation during colon tumorigenesis. Cancer Res, 69, 3382-9.

Ibrahim A, Khaled H, Mikhail N, et al (2014). Cancer incidence in Egypt, results of the national population-based cancer registry program. J Cancer Epidemiology.

Ieni A, Giuffre G, Adamo V, et al (2011). Prognostic impact of CD133 immunoexpression in node-negative invasive breast carcinomas. Anticancer Res, 31, 1315-20.

Jiang F, Qiu Q, Khanna A, et al (2009). Aldehyde dehydrogenase 1 is a tumor stem cell-associated marker in lung cancer. $\mathrm{Mol}$ Cancer Res, 7, 330-8.

Keymoosi H, Gheytanchi E, Asgari M, et al (2014). ALDH1 in combination with CD44 as putative cancer stem cell markers are correlated with poor prognosis in urothelial carcinoma of the urinary bladder. Asian Pac J Cancer Prev, 15, 2013-20.

Lamy PJ, Martin PM, Romieu G, et al (2013). Clinical validity/utility, change in practice patterns, and economic implications of risk stratifiers to predict outcomes for earlystage breast cancer, a systematic review. J Natl Cancer Inst, $105,149$.

Liao Y, Hu X, Huang X, et al (2010). Quantitative analyses of CD133 expression facilitate researches on tumor stem cells. Biological \& Pharmaceutical Bulletin, 33, 738-742.

Madjd Z, Ramezani B, Molanae S, et al (2012). High Expression of stem cell marker ALDH1 is associated with reduced BRCA1 in invasive breast carcinomas. Asian Pac J Cancer Prev, 13, 2973-8.

Meacham C, and Morrison S (2013), Tumor heterogeneity and cancer cell plasticity. Nature, 501, 328-37.

Meyer M, Fleming J, Ali M, et al (2009). Dynamic regulation of CD24 and the invasive, CD44posCD24neg phenotype in breast cancer cell lines. Breast Cancer Res 11, R82.

Mills J, and Shivdasani R (2011). Gastric epithelial stem cells. Gastroenterology, 140, 412-24.

Morimoto K, Kim S, Tanei T, et al (2009). Stem cell marker aldehyde dehydrogenase 1-positive breast cancers are characterized by negative estrogen receptor, positive human epidermal growth factor receptor type 2, and high Ki67 expression. Cancer Sci, 100, 1062-8.

Navin N, Kendall J, Troge J, et al (2011). Tumor evolution inferred by single-cell sequencing. Nature, 472, 90-4.

Ohi Y, Umekita Y, Yoshioka T, et al (2011). Aldehyde dehydrogenase 1 expression predicts poor prognosis in triple-negative breast cancer. Histopathology, 59, 776-80

Park SY, Lee HE, Li H, et al (2010). Heterogeneity for stem cellrelated markers according to tumor subtype and histologic stage in breast cancer. Clin Cancer Res, 16, 876-87.

Rao Q, Yao T, Zhang B, et al (2012). Expression and functional role of ALDH1 in cervical carcinoma cells. Asian Pac J Cancer Prev, 13, 1325-31.

Rasha M Abd El Atti, Riham M Abu-Zeid (2012). CD133 and FGF7. Egyptian Journal of Pathology, 32, 142-9.

Resetkova E, Reis-Filho J, Jain R, et al (2010). Prognostic impact of ALDH1 in breast cancer, a story of stem cells and tumor microenvironment. Breast Cancer Res Treat, 123, 97-108. 
Reya T, Morrison S, Clarke M, et al (2001). Stem cells, cancer, and cancer stem cells. Nature, 414, 105-11.

Sakakibara M, Fujimori T, Miyoshi T, et al (2012). Aldehyde dehydrogenase 1-positive cells in axillary lymph node metastases after chemotherapy as a prognostic factor in patients with lymph node-positive breast cancer. Cancer, 118, 3899-910.

Saricanbaz I, Karahacioglu E, Ekinci O, et al (2014). Prognostic significance of expression of CD133 and Ki-67 in gastric cancer. Asian Pac J Cancer Prev, 15, 8215-9.

Satpute P, Hazarey V, Ahmed R et al (2013). Cancer stem cells in head and neck squamous cell carcinoma, a review. Asian Pac J Cancer Prev, 14, 5579-87.

Schneider M,Huber J, Hadaschik B, et al (2012). Characterization of colon cancer cells, a functional approach characterizing CD133 as a potential stem cell marker," BMC Cancer, 12, 96 .

Shi C, Tian R, Wang M, et al (2010). CD44+ CD133+ population exhibits cancer stem cell-like characteristics in human gallbladder carcinoma. Cancer Biol Ther, 10, 1182-90.

Shimada Y, Ishii G, Nagai K et al (2009). Expression of podoplanin, CD44, and $\mathrm{p} 63$ in squamous cell carcinoma of the lung. Cancer Sci, 100, 2054-9.

Tessari A, Palmieri D, Di Cosimo S (2013). Overview of diagnostic/targeted treatment combinations in personalized medicine for breast cancer patients. Pharmgenomics Pers Med, 7, 1-19.

Timothy E, Wang D, Harki D (2013). Cell surface markers of cancer stem cells, diagnostic macromolecules and targets for drug delivery. Drug Delivery and Translational Research, 3, 121-42.

Tirino V, Camerlingo R, Franco R, et al (2009). The role of CD133 in the identification and characterisation of tumour-initiating cells in non-small-cell lung cancer. Eur $J$ Cardiothorac Surg, 36, 446-53.

Turner N, Pestrin M, Galardi F, et al (2014). Can biomarker assessment on circulating tumor cells help direct therapy in metastatic breast cancer? Cancers (Basel), 6, 684-707.

Visvader J, and Lindeman G (2008). Cancer stem cells in solid tumours, Accumulating evidence and unresolved questions. Nat Rev Cancer, 8, 755-68.

Wang K, Liu L, Zhang T, et al (2011). Oxaliplatin-incorporated micelles eliminate both cancer stem-like and bulk cell populations in colorectal cancer. Int J Nanomedicine, $\mathbf{6}$, 3207-18.

Wright M, Calcagno A, Salcido C, et al (2008). Brca1 breast tumors contain distinct CD44+/CD24- and CD133+ cells with cancer stem cell characteristics. Breast Cancer Res, 10, 10.

Wu Y, and Wu P (2009). CD133 as a marker for cancer stem cells, progresses and concerns. Stem Cells Dev, 18, 1127-34.

Yan X, Ma L, Yi D, et al (2011). A CD133-related gene expression signature identifies an aggressive glioblastoma subtype with excessive mutations. Proc Natl Acad Sci U S A, 108, 1591-6.

Zhao P, Lu Y, Jiang X, et al (2011). Clinicopathological significance and prognostic value of CD133 expression in triple-negative breast carcinoma. Cancer Sci, 102, 1107-11.

Zobalova R, Prokopova K, Stantic M, et al (2011). The Potential role of CD133 in immune surveillance and apoptosis, a mitochondrial connection? Antioxidants \& Redox Signaling, 15, 2989-3002. 